\title{
Evaluation of the Impact of an Institution-Specific Dofetilide Initiation Protocol on Mean Hospital Length of Stay and Cost for Dofetilide Initiation
}

\author{
Steven P. Kennedy ${ }^{1}$ (D) Hanna Iaizzo $^{2} \cdot$ Evgueni Fayn $^{1} \cdot$ Dalip Singh $^{1}$
}

Published online: 18 April 2018

(C) The Author(s) 2018

\begin{abstract}
Background Dofetilide is a class III antiarrhythmic drug indicated for the conversion and maintenance of normal sinus rhythm in symptomatic patients with atrial fibrillation/atrial flutter. Delay in initiation of dofetilide therapy may increase the duration of hospitalization from two nights to three nights to complete US Food and Drug Administration-required monitoring. Therefore, substantial cost savings may be associated with implementation of an institution-specific dofetilide initiation protocol in order to reduce hospitalization to two nights. This could potentially be achieved through protocol-defined utilization of the option for a condensed dosing interval for the first three doses of dofetilide in order to ensure the administration of two doses on the first day of hospitalization.

Objective The primary objective of this study was to assess the impact of an institution-specific dofetilide initiation protocol on mean hospital length of stay and cost for dofetilide initiation.

Methods The study design was a retrospective review of 150 patients admitted to the Clement J. Zablocki Veterans Affairs Medical Center for the purpose of dofetilide initiation. Matching time periods of 18 months before and after implementation of the institution-specific dofetilide initiation protocol were used to randomly select 75 patients from each time period for comparison.
\end{abstract}

Steven P. Kennedy

steven.kennedy@va.gov

1 Clement J. Zablocki VA Medical Center, 5000 W. National Ave, Milwaukee, WI 53295, USA

2 Hayat Pharmacy, 1919 W. North Ave, Milwaukee, WI 53205, USA
Results A significant reduction in mean hospital length of stay of 0.56 nights post-implementation of the institutionspecific dofetilide initiation protocol was identified $(95 \%$ confidence interval $0.20-0.92 ; P=0.0029)$. Assuming hospital adjusted expenses per inpatient day of US\$1831-2413, a reduction in hospital length of stay of 0.56 nights resulted in estimated cost savings of US\$1025-1351 per admission for dofetilide initiation.

Conclusion Implementation of an institution-specific dofetilide initiation protocol decreases mean hospital length of stay and cost for dofetilide initiation.

\section{Key Points for Decision Makers}

Mean hospital length of stay and cost for dofetilide initiation can be minimized through implementation of an institution-specific dofetilide initiation protocol that facilitates timely administration of the first dose of dofetilide so that only two nights of hospitalization are required to complete monitoring for the first five doses as required by the US Food and Drug Administration.

The possibility of a condensed dosing interval for dofetilide after implementation of the institutionspecific dofetilide initiation protocol (dosing as frequent as every $9 \mathrm{~h}$ for doses one through three when the first dose is administered as late as 3 p.m. on the first day of hospitalization) does not increase the frequency of dofetilide dose reduction or dofetilide treatment failure. 


\section{Introduction}

\subsection{Background}

Atrial fibrillation is the most common type of arrhythmia, affecting nearly $1 \%$ of the general population [1]. In symptomatic patients with atrial fibrillation, antiarrhythmic therapy can be useful to achieve and maintain sinus rhythm in order to alleviate symptoms and improve heart failure management [2-4]. Dofetilide is a class III antiarrhythmic drug indicated for the conversion and maintenance of normal sinus rhythm in symptomatic patients with atrial fibrillation/atrial flutter [5]. Dofetilide, in a dose-dependent manner, prolongs both atrial and ventricular repolarization and, therefore, increases the refractory period within the cardiac myocyte. An increase in QT interval associated with dofetilide-induced prolongation of the refractory period can cause serious ventricular arrhythmias including torsades de pointes (TdP). Based on a recent review of published literature, TdP is estimated to occur at a rate of $2.4 \%$ amongst patients who are prescribed dofetilide [6].

Because of the risk for QT prolongation and TdP, the US Food and Drug Administration (FDA) mandates that therapy with dofetilide be initiated (and, if necessary, reinitiated) in a controlled setting for a minimum of 3 days (five doses of dofetilide) [5]. Since a controlled setting for dofetilide initiation typically necessitates two to three nights of hospitalization, there is substantial monetary cost associated with initiating therapy. According to the latest statistics from Kaiser State Health Facts, hospital adjusted expenses per inpatient day in the United States ranged from US\$1831 to US\$2413 in 2015 [7]. Assuming a minimum hospital length of stay of two nights for dofetilide initiation, this corresponds to a total cost of hospitalization of US\$3662-4826.

At the Clement J. Zablocki Veterans Affairs (VA) Medical Center, a majority of patients started on dofetilide are scheduled hospital admissions for the purpose of initiating the medication. Historically, no institution-specific protocol was in place to guide dofetilide initiation. However, all prescribers were expected to be in compliance with manufacturer-imposed regulations for dofetilide initiation. Although no concerns with regulation compliance were identified despite the absence of an institutionspecific protocol, delays in initiation of therapy occurred frequently and often resulted in patients receiving only their evening dose of dofetilide instead of two doses on the first day of hospitalization. Consequently, delays of this nature frequently increased hospital length of stay by at least one night and estimated total cost of hospitalization by at least US\$1831-2413 [7].
To address this inefficiency, an institution-specific dofetilide initiation protocol was developed and implemented in May of 2015. The protocol conformed to all manufacturer-imposed regulations and included an additional component designed to facilitate the administration of two doses of dofetilide on the first day of hospitalization (Fig. 1). This was achieved through protocol authorization for the cardiology pharmacist to schedule a "first dose now" upon receiving any dofetilide formulary-restricted request after the standard morning administration time of 9 a.m. for medications dosed every $12 \mathrm{~h}$. Additionally, the protocol allows for the pharmacist to schedule a second dose of dofetilide to be given on the first day of hospitalization as long as the administration time is at least $9 \mathrm{~h}$ after the first dose is given. A condensed dosing interval of this nature effectively allows for patients to receive two doses of dofetilide on the first day of hospitalization as long as the first dose is administered by 3 p.m. (with the second dose administered at 12 a.m. and subsequent doses administered on the standard 9 a.m. -9 p.m. schedule), and therefore, may reduce hospital length of stay and cost. It was hypothesized that implementation of this institutionspecific dofetilide initiation protocol would decrease mean hospital length of stay and cost without increasing the frequency of dofetilide dose reduction (i.e., QT prolongation), TdP, or other adverse events resulting in discontinuation of therapy.

\subsection{Objectives}

The primary objective of this study was to assess the impact of an institution-specific dofetilide initiation protocol on mean hospital length of stay and cost for dofetilide initiation.

\section{Methods}

\subsection{Setting}

The Clement J. Zablocki VA Medical Center is a tertiary care and academic medical center located in Milwaukee, Wisconsin that serves the Veteran population of Southeastern Wisconsin. The medical center delivers primary, secondary, and tertiary medical care in 168 acute care operating beds and provides over 500,000 visits annually through an extensive outpatient program [8]. Included in the 168 acute care beds is a 15-bed cardiology unit managed by a dedicated inpatient cardiology service that admits approximately 50-75 patients per year for the purpose of dofetilide initiation. 


\begin{tabular}{|c|c|c|c|c|c|c|c|c|c|c|c|c|c|c|c|c|}
\hline Hospital Day & \multicolumn{4}{|c|}{1} & \multicolumn{4}{|c|}{2} & \multicolumn{4}{|c|}{3} & \multicolumn{4}{|c|}{4} \\
\hline Time & 9am & $12 \mathrm{pm}$ & $3 \mathrm{pm}$ & $9 \mathrm{pm}$ & 12am & $9 \mathrm{am}$ & $12 \mathrm{pm}$ & $9 \mathrm{pm}$ & $12 \mathrm{am}$ & 9am & $12 \mathrm{pm}$ & $9 \mathrm{pm}$ & 12am & 9am & $12 \mathrm{pm}$ & $9 \mathrm{pm}$ \\
\hline $\begin{array}{c}\text { Pre-protocol } \\
\text { implementation }\end{array}$ & & $\underset{\begin{array}{c}\text { Late } \\
\text { Admission }\end{array}}{\boldsymbol{\Delta}}$ & & $\underset{\substack{\text { Dose } \\
\# 1}}{\boldsymbol{\Delta}}$ & & $\underset{\substack{\text { Dose } \\
\# 2}}{\Delta}$ & & $\underset{\substack{\text { Dose } \\
\# 3}}{\boldsymbol{\Delta}}$ & & $\underset{\substack{\text { Dose } \\
\# 4}}{\boldsymbol{\Delta}}$ & & $\underset{\substack{\text { Dose } \\
\# 5}}{\boldsymbol{\Delta}}$ & $\begin{array}{r}\text { Last } \mathrm{E} \\
\text { hospi } \\
\mathrm{h}\end{array}$ & $\begin{array}{l}G \text { at } 11_{f} \\
\text { day } 3= \\
\text { pital day } \\
\text { hospit }\end{array}$ & $\begin{array}{l}\mathrm{m} \text { after } 5^{\text {th }} \\
\text { no dischar } \\
4 \text { (3 nights } \\
\text { lization) }\end{array}$ & $\begin{array}{l}\text { dose on } \\
\text { se until } \\
\text { of }\end{array}$ \\
\hline $\begin{array}{l}\text { Post-protocol } \\
\text { implementation }\end{array}$ & & $\underset{\begin{array}{c}\text { Late } \\
\text { Admission }\end{array}}{\boldsymbol{\Delta}}$ & $\underset{\substack{\text { Dose } \\
\# 1}}{\boldsymbol{\Delta}}$ & & $\underset{\substack{\text { Dose } \\
\# 2}}{\boldsymbol{\Delta}}$ & $\underset{\substack{\text { Dose } \\
\# 3}}{\boldsymbol{\Delta}}$ & & $\underset{\substack{\text { Dose } \\
\# 4}}{\boldsymbol{\Delta}}$ & & $\underset{\substack{\text { Dose } \\
\# 5}}{\boldsymbol{\Delta}}$ & & & $\begin{array}{r}\text { Disch } \\
\text { after } \\
\quad n\end{array}$ & $\begin{array}{l}\text { rge after } \\
\text { "ndose or } \\
\text { thts of h }\end{array}$ & $\begin{array}{l}\text { last EKG a } \\
\text { hospital d } \\
\text { sppitalizatic }\end{array}$ & $\begin{array}{l}11 \text { am } \\
\text { n) } 3(2\end{array}$ \\
\hline
\end{tabular}

Fig. 1 Dofetilide administration schedule pre- and post-implementation of the institution-specific initiation protocol. $E K G$ electrocardiogram

\subsection{Design}

The study design was a retrospective review of 150 patients admitted to the Clement J. Zablocki VA Medical Center for the purpose of dofetilide initiation. Matching time periods of 18 months before and after implementation of the institution-specific dofetilide initiation protocol were used to randomly select 75 patients from each time period for comparison. The study was determined exempt from the Clement J. Zablocki VA Medical Center Institutional Review Board approval requirements because of the retrospective chart review design and collection of data without identifiers or a link to identifiers.

\subsection{Criteria}

Inclusion criteria for this study included all Clement $\mathrm{J}$. Zablocki VA Medical Center patients admitted for dofetilide initiation or re-initiation between December 2013 and November 2016. Exclusion criteria for this study included all patients with an inpatient dofetilide medication order during the study period who were taking dofetilide prior to hospitalization. No patients were excluded from chart review on the basis of co-morbidities or for any other reason. It was the responsibility of the prescribing physician to select appropriate patients for dofetilide use pre- and postprotocol implementation (i.e., avoid dofetilide use in patients with congenital long QT syndrome or prolonged baseline QT, severe left ventricular hypertrophy, creatinine clearance less than $20 \mathrm{~mL} / \mathrm{min}$, or concurrent use of contraindicated medications). Therefore, any patient admitted for dofetilide initiation was considered for review in order to obtain the most accurate "real world" representation of dofetilide tolerability pre- and post-protocol implementation.

\subsection{Enrollment}

Data from the electronic medical record were used to generate a list of 344 Clement J. Zablocki VA Medical Center patients who had an inpatient dofetilide medication order between December 2013 and November 2016. Randomly selected samples of 75 patients admitted pre- and post-implementation of the institution-specific dofetilide initiation protocol were used for comparison.

\subsection{Procedures}

Chart review was performed on each randomly selected patient admitted for dofetilide initiation pre- or post-implementation of the institution-specific dofetilide initiation protocol. Data collected for each patient included the following: age, sex, body weight, calculated creatinine clearance at the time of dofetilide initiation (CockcroftGault method using ideal body weight), ejection fraction, presence of co-morbidities (heart failure with reduced ejection fraction, coronary artery disease, hypertension, diabetes mellitus), baseline potassium level, baseline magnesium level, baseline electrocardiogram data (QTc interval, QRS interval, heart rate), concurrent use of loop diuretics, concurrent use of QT-prolonging medications, dofetilide starting dose, dofetilide dose upon discharge, number of dofetilide dose reductions, and number of nights of hospitalization required to complete dofetilide initiation. Additionally, for cases where dofetilide was discontinued prior to discharge (i.e., treatment failure), the reason for discontinuation was recorded (i.e., discontinuation because of QT prolongation despite two dose reductions, TdP, or other adverse event resulting in discontinuation of therapy). Although the manufacturer recommends use of actual body weight when calculating creatinine clearance for determining the initial dofetilide dose, ideal body weight was used as per electrophysiologist preference at our institution. The use of ideal body weight is preferred because of concerns with overestimation of renal function and dofetilide overdosing when using actual body weight for calculating creatinine clearance in the predominately obese and elderly population at our institution. 


\subsection{Outcomes Measured}

The primary outcome measure was the difference in mean hospital length of stay pre- and post-implementation of the institution-specific dofetilide initiation protocol. Secondary outcome measures included comparisons of the differences in frequency of dofetilide dose reduction and adverse events resulting in discontinuation of therapy pre- versus post-implementation of the institution-specific dofetilide initiation protocol.

\subsection{Statistical Analysis}

Student's $t$ tests were conducted to compare the difference in patient baseline characteristics (ejection fraction, potassium level, magnesium level, QTc, QRS, and heart rate) and mean hospital length of stay pre- and post-implementation of the institution-specific dofetilide initiation protocol. Chi-squared tests (or Fischer's exact tests if cells with an expected value of less than five were present) were conducted to determine if any differences in patient baseline characteristics (frequency of co-morbidities, concurrent use of loop diuretics, concurrent use of QT-prolonging medications) and the frequency of dofetilide dose reduction or treatment failure were statistically significant. All data analyses were performed using Microsoft Excel, version 2007 (Microsoft Corp., Redmond, WA, USA). Results were considered significant at a two-tailed $P$ value of less than 0.05 .

\section{Results}

A majority of patients $(n=145,96.7 \%)$ included for chart review were male (Table 1). The mean age was $69.5 \pm 8.9$ years old, and the study population was predominately obese, with a mean body mass index of $32.9 \pm 6.9 \mathrm{~kg} / \mathrm{m}^{2}$. The mean creatinine clearance at time of dofetilide initiation was $69.1 \pm 24.6 \mathrm{~mL} / \mathrm{min}$. The mean left ventricular ejection fraction was $46.4 \pm 12.7 \%$, and 45 patients (30\%) had heart failure with reduced ejection fraction. Additional cardiovascular co-morbidities were present and included coronary artery disease $(n=78$, $52 \%)$, hypertension $(n=126,84 \%)$, and diabetes mellitus $(n=70,46.7 \%)$. The mean QTc at baseline was $457 \pm 33 \mathrm{~ms}$, and 35 patients $(23.3 \%)$ were taking concomitant QT-prolonging medications at the time of dofetilide initiation. No statistically significant differences between groups were observed for any of the patient baseline characteristics, with the exception of baseline potassium and magnesium levels. Although the mean baseline potassium level was significantly lower in the post-protocol implementation group (4.07 vs. $4.21 \mathrm{mEq} / \mathrm{L}$;
$P=0.03)$ and the mean baseline magnesium level was significantly higher in the post-protocol implementation group ( 2.03 vs. $1.91 \mathrm{mEq} / \mathrm{L} ; P=0.001)$, these differences were not considered to be clinically significant.

The dofetilide starting dose was commensurate with manufacturer-recommended dosing for renal function in 64 patients $(85.3 \%)$ in the pre-protocol implementation group and 65 patients $(86.7 \%)$ in the post-protocol implementation group. This $1.4 \%$ difference between groups was not statistically significant $(P=0.8155)$. Of the 21 patients that received a dofetilide starting dose that was not commensurate with manufacturer-recommended dosing for renal function, the majority $(n=18,85.7 \%)$ were under-dosed. This was typically a result of provider discretion with consideration of baseline risk factors for QTc prolongation, including advanced age, borderline QTc, or borderline renal function.

Mean hospital length of stay was 2.91 nights in the preprotocol implementation group and 2.35 nights in the postprotocol implementation group (Table 2). For the primary outcome measure, there was a statistically significant reduction in mean hospital length of stay of 0.56 nights post-protocol implementation $(95 \%$ confidence interval 0.20-0.92; $\quad P=0.0029$ ). Assuming hospital adjusted expenses per inpatient day of US\$1831-2413 in 2015 [7], a reduction in hospital length of stay of 0.56 nights resulted in estimated cost savings of US\$1025-1351 per admission for dofetilide initiation after protocol implementation.

A total of 53 patients (35.3\%), including pre- and postprotocol implementation, required at least one dofetilide dose reduction because of QT prolongation (Table 3). Of these patients that required dofetilide dose reduction, a majority $(n=48,91 \%)$ required a single dose reduction compared to five patients $(9 \%)$ that required two dose reductions. For the secondary outcome measure of frequency of dofetilide dose reduction, there was no statistically significant difference in the number of patients that required a single dose reduction pre- versus post-protocol implementation (28 vs. $36 \%$, pre- vs. post-protocol, respectively; $P=0.2968$ ) or two dose reductions preversus post-protocol implementation (2.7 vs. $4 \%$, pre- vs. post-protocol, respectively; $P=1$ ).

Dofetilide treatment failures because of TdP, QT prolongation after two dose reductions, or other adverse events resulting in discontinuation of therapy were infrequent overall ( $n=13,8.7 \%)$, with no statistically significant difference observed in the frequency of treatment failure pre- versus post-protocol implementation $(9.3$ vs. $8 \%$, prevs. post-protocol, respectively; $P=0.7735$ ) (Table 4). There were two occurrences of $\mathrm{TdP}$ in the total study population $(1.3 \%)$, including one $(1.3 \%)$ in the pre-protocol implementation group and one $(1.3 \%)$ in the postprotocol implementation group. The episode of TdP in the 
Table 1 Study population baseline characteristics

\begin{tabular}{|c|c|c|c|c|}
\hline & All patients $(n=150)$ & Pre-implementation $(n=75)$ & Post-implementation $(n=75)$ & $P$ value* \\
\hline Female & $5(3.3 \%)$ & $3(4 \%)$ & $2(2.7 \%)$ & 0.6411 \\
\hline Male & $145(96.7 \%)$ & $72(96 \%)$ & $73(97.3 \%)$ & 0.6518 \\
\hline Age (years) & $69.5 \pm 8.9$ & $68.9 \pm 9.4$ & $70.2 \pm 8.5$ & 0.3823 \\
\hline Body mass index $\left(\mathrm{kg} / \mathrm{m}^{2}\right)$ & $32.9 \pm 6.9$ & $33.0 \pm 7.2$ & $32.8 \pm 6.6$ & 0.8482 \\
\hline Creatinine clearance $^{\mathrm{a}}(\mathrm{mL} / \mathrm{min})$ & $69.1 \pm 24.6$ & $68.4 \pm 24.4$ & $69.9 \pm 24.8$ & 0.7052 \\
\hline Ejection fraction $(\%)$ & $46.4 \pm 12.7$ & $45.9 \pm 13.0$ & $46.8 \pm 12.6$ & 0.6677 \\
\hline Heart failure (ejection fraction $\leq 40 \%$ ) & $45(30 \%)$ & $25(33.3 \%)$ & $20(26.7 \%)$ & 0.3729 \\
\hline Coronary artery disease & $78(52 \%)$ & $37(49.3 \%)$ & $41(54.7 \%)$ & 0.5133 \\
\hline Hypertension & $126(84 \%)$ & $62(82.3 \%)$ & $64(85.3 \%)$ & 0.6560 \\
\hline Diabetes mellitus & $70(46.7 \%)$ & $36(48 \%)$ & $34(45.3 \%)$ & 0.7434 \\
\hline Potassium (mEq/L) & $4.14 \pm 0.4$ & $4.21 \pm 0.39$ & $4.07 \pm 0.39$ & 0.0338 \\
\hline Magnesium (mEq/L) & $1.97 \pm 0.23$ & $1.91 \pm 0.22$ & $2.03 \pm 0.23$ & 0.0012 \\
\hline QTc (ms) & $457 \pm 33$ & $454 \pm 33$ & $461 \pm 33$ & 0.2442 \\
\hline QRS (ms) & $115 \pm 32$ & $116 \pm 32$ & $114 \pm 31$ & 0.7453 \\
\hline Ventricular rate & $76 \pm 18$ & $75 \pm 18$ & $78 \pm 18$ & 0.4430 \\
\hline Concurrent loop diuretic use & $68(45.3 \%)$ & $34(45.3 \%)$ & $34(45.3 \%)$ & 1 \\
\hline 1 concurrent QT-prolonging medication & $25(16.7 \%)$ & $14(18.7 \%)$ & $11(14.7 \%)$ & 0.5110 \\
\hline 2 concurrent QT-prolonging medications & $9(6 \%)$ & $4(5.3 \%)$ & $5(6.7 \%)$ & 1 \\
\hline 3 concurrent QT-prolonging medications & $1(0.7 \%)$ & $0(0 \%)$ & $1(1.3 \%)$ & 1 \\
\hline
\end{tabular}

Values are mean \pm standard deviation or $n(\%)$

*Two-tailed Student's $t$ test or Chi-squared test or Fischer's exact test if cells with expected value of $<5$ were present, significance level of $p<0.05$

${ }^{\mathrm{a}}$ Cockcroft-Gault method using ideal body weight

Table 2 Number of nights of hospitalization pre- and postprotocol implementation

\begin{tabular}{lll}
\hline & Pre-implementation $(n=75)$ & Post-implementation $(n=75)$ \\
\hline Number of nights & $2.91 \pm 1.43$ & $2.35 \pm 0.73$ \\
Difference & 0.56 & \\
95\% confidence interval & $0.20-0.92$ & \\
$P$ value* & 0.0029 & \\
\hline
\end{tabular}

Values are mean \pm standard deviation

*Two-tailed Student's $t$ test (statistical power $=85.6 \%$ )

Table 3 Frequency of dofetilide dose reduction

\begin{tabular}{lcccc}
\hline & All patients $(n=150)$ & Pre-implementation $(n=75)$ & Post-implementation $(n=75) P$ value \\
\hline Single dose reduction & $48(32 \%)$ & $21(28 \%)$ & $27(36 \%)$ & 0.2968 \\
Two dose reductions & $5(3.3 \%)$ & $2(2.7 \%)$ & $3(4 \%)$ & 1 \\
Total requiring $\geq 1$ dose reduction & $53(35.3 \%)$ & $23(30.7 \%)$ & $30(40 \%)$ & 0.2388 \\
\hline
\end{tabular}

Dofetilide dose reduction is equivalent to a QT prolongation event

${ }^{\dagger}$ Chi-squared test or Fischer's exact test if cells with expected value of $<5$ were present, significance level of $p<0.05$

post-protocol implementation group occurred approximately $2 \mathrm{~h}$ before the fourth scheduled dose of dofetilide and was determined to be caused by the superimposition of an ectopic beat on the $\mathrm{T}$ wave of the preceding beat (i.e., $\mathrm{R}$-on- $\mathrm{T}$ phenomenon) in the setting of sinus bradycardia and frequent premature ventricular contractions. Although 
Table 4 Frequency of dofetilide treatment failure

\begin{tabular}{|c|c|c|c|c|}
\hline & $\begin{array}{l}\text { All patients } \\
(n=150)\end{array}$ & $\begin{array}{l}\text { Pre-implementation } \\
(n=75)\end{array}$ & $\begin{array}{l}\text { Post-implementation } \\
(n=75)\end{array}$ & $P$ value $^{\dagger}$ \\
\hline Torsade de pointes & $2(1.3 \%)$ & $1(1.3 \%)$ & $1(1.3 \%)$ & 1 \\
\hline QT prolongation after 2 dose reductions & $3(2 \%)$ & $1(1.3 \%)$ & $2(2.7 \%)$ & 1 \\
\hline $\begin{array}{l}\text { Other adverse event resulting in therapy } \\
\text { discontinuation }\end{array}$ & $8(5.3 \%)$ & $5(6.7 \%)$ & $3(4 \%)$ & 0.719 \\
\hline Total treatment failures & $13(8.7 \%)$ & $7(9.3 \%)$ & $6(8 \%)$ & 0.7735 \\
\hline
\end{tabular}

${ }^{\dagger}$ Chi-squared test or Fischer's exact test if cell(s) with expected value of $<5$ were present, significance level of $p<0.05$

a condensed dosing interval for the first three doses of dofetilide was utilized as described previously (Fig. 1), this variable was not thought to contribute to the TdP episode because the QTc interval was normal $(429 \mathrm{~ms}$ in sinus rhythm) prior to the event and did not prolong from baseline (baseline of $458 \mathrm{~ms}$ in atrial fibrillation) after any of the first three doses of dofetilide.

All other instances of dofetilide discontinuation in the total study population occurred because of QT prolongation after two dose reductions $(n=3,2 \%)$ or other adverse events resulting in therapy discontinuation $(n=8,5.3 \%)$. There was no statistically significant difference in the frequency of QT prolongation after two dose reductions preversus post-protocol implementation (1.3 vs. $2.7 \%$, pre- vs. post-protocol, respectively; $P=1$ ) or other adverse events resulting in therapy discontinuation pre- versus post-protocol implementation (6.7 vs. $4 \%$, pre- vs. post-protocol, respectively; $P=0.719$ ). Other adverse events resulting in therapy discontinuation included failure to maintain sinus rhythm after completion of dofetilide initiation or the occurrence of non-sustained ventricular tachycardia or sinus pauses after starting dofetilide.

\section{Discussion}

The cost of hospitalization for dofetilide initiation can be minimized by timely administration of the first dose of dofetilide so that only two nights of hospitalization are required to complete monitoring for the first five doses as required by the FDA. With consideration of the 2.91 average nights of hospitalization in the pre-protocol implementation group, it is evident that many patients required more than two nights of hospitalization to complete dofetilide initiation prior to protocol implementation. This was a frequent observation before development of the institution-specific dofetilide initiation protocol and was usually a result of only one dose of dofetilide administered on the first day of hospitalization because of hospital admission well after the standard morning administration time of 9 a.m. for medications dosed every $12 \mathrm{~h}$. This inefficiency served as the impetus for creation of the institution-specific dofetilide initiation protocol. The present study was conducted to test the hypothesis that protocol-defined utilization of the option for a condensed dosing interval for the first three doses of dofetilide in order to ensure the administration of two doses on the first day of hospitalization would decrease mean hospital length of stay and cost without increasing the frequency of dofetilide dose reduction or other adverse events resulting in discontinuation of therapy. The statistically significant reduction of 0.56 nights of hospitalization post-protocol implementation confirms the hypothesis that the duration of hospitalization would decrease after implementation of the protocol and demonstrates substantial value in the form of estimated cost savings of US\$1025-1351 per admission for dofetilide initiation. With a conservative estimate of 50 admissions per year at the Clement J. Zablocki VA Medical Center for the purpose of dofetilide initiation, the institution-specific dofetilide initiation protocol results in annual estimated cost savings of US\$51,250-67,550 for the medical center.

The possibility of a condensed dosing interval for dofetilide after implementation of the institution-specific dofetilide initiation protocol (dosing as frequent as every $9 \mathrm{~h}$ for doses one through three when the first dose was administered as late as 3 p.m. on the first day of hospitalization) was a potential concern for causing an increase in the frequency of dofetilide dose reduction and dofetilide treatment failure post-protocol implementation. Therefore, the secondary objective of this study was to compare the frequency of dofetilide dose reduction and treatment failure pre- versus post-protocol implementation. Again, the study results confirmed the hypothesis that implementation of the institution-specific dofetilide initiation protocol would not increase the frequency of dofetilide dose reduction or other adverse events related to the potential for a condensed dosing interval, as there were no statistically significant differences in the frequency of dofetilide dose reduction, $\mathrm{TdP}$, or other adverse events resulting in treatment failure 
pre- versus post-protocol implementation. Additionally, the frequency of TdP and dofetilide treatment failure identified in this review is consistent with what has been previously reported in the literature [9-15]. These results alleviate potential concern that the cost savings associated with a reduced duration of hospitalization for dofetilide initiation through utilization of a condensed dosing interval would be realized at the expense of an increase in the frequency of QT prolongation, TdP, or other adverse events resulting in discontinuation of therapy.

\subsection{Limitations}

The authors acknowledge limitations in the study design that may decrease the validity of the results. The internal validity of the study results may be decreased by the relatively small sample size. Although the study was adequately powered (statistical power $=85.6 \%$ ) to detect a significant difference in the mean duration of hospitalization pre- and post-implementation of the institution-specific dofetilide initiation protocol, a longer sampling time frame for chart review and, consequently, a larger sample size would be desirable for further evaluation of the significance of differences in the frequency of dofetilide dose reduction and treatment failure pre- and post-implementation of the protocol.

The external validity of the study results may be decreased because the investigation was conducted at a single VA medical center consisting of a patient population that is predominantly male. The small number of female patients included for review (only $3.3 \%$ of the study population) is consistent with the VA patient population as a whole, but may not be representative of patient populations treated at other medical centers. Therefore, the study results should be interpreted with caution when applied to patient populations with larger percentages of female patients, as mean QTc is longer in females than in males and longer baseline QTc may decrease dofetilide tolerability.

Additionally, the external validity of the study results may be decreased because of the conservative nature of initial dofetilide dosing utilized at our medical center. Although the manufacturer recommends use of actual body weight when calculating creatinine clearance for determining the initial dofetilide dose, ideal body weight was used as per electrophysiologist preference at our institution. The use of ideal body weight for calculating creatinine clearance in the predominately obese patient population at our institution results in a relatively conservative estimate of renal function and, consequently, increases the probability of lower initial dofetilide dosing. Other institutions with predominately obese patient populations that utilize actual body weight when calculating creatinine clearance for determining dofetilide dosing may result in more aggressive initial dosing and, consequently, increased probability of dofetilide dose reduction and/or treatment failure.

\section{Conclusion}

Implementation of an institution-specific dofetilide initiation protocol utilizing the option for a condensed dosing interval for the first three doses of dofetilide in order to ensure the administration of two doses on the first day of hospitalization decreases mean hospital length of stay and cost of hospitalization without increasing the frequency of dofetilide dose reduction or other adverse events resulting in discontinuation of therapy. Medical centers that admit patients for the purpose of dofetilide initiation should consider implementation of this approach in order to reduce the cost burden associated with initiation of dofetilide therapy.

Author Contributions All authors of this manuscript made substantial contributions to the conception and design, acquisition of data, and analysis and interpretation of data. All authors participated in drafting the manuscript and revising it critically for important intellectual content and gave final approval of the version to be submitted and any revised version.

Data Availability Statement The datasets generated and analyzed during the current study are available from the corresponding author on reasonable request.

\section{Compliance with Ethical Standards}

This research received no specific grant from any funding agency in the public, commercial, or not-for-profit sectors. The authors (Dr. Kennedy, Dr. Iaizzo, Dr. Fayn, and Dr. Singh) certify that they have no affiliations with or involvement in any organization or entity with any financial interest or non-financial interest in the subject matter or materials discussed in this article. This study was determined exempt from the Clement J. Zablocki VA Medical Center Institutional Review Board approval requirements because of the retrospective chart review design and collection of data without identifiers or a link to identifiers. This study has been performed in accordance with the ethical standards of the Declaration of Helsinki.

Open Access This article is distributed under the terms of the Creative Commons Attribution-NonCommercial 4.0 International License (http://creativecommons.org/licenses/by-nc/4.0/), which permits any noncommercial use, distribution, and reproduction in any medium, provided you give appropriate credit to the original author(s) and the source, provide a link to the Creative Commons license, and indicate if changes were made. 


\section{References}

1. Go AS, Hylek EM, Phillips KA, et al. Prevalence of diagnosed atrial fibrillation in adults: national implications for rhythm management and stroke prevention: the AnTicoagulation and Risk Factors in Atrial Fibrillation (ATRIA) Study. JAMA. 2001;285:2370-5.

2. Van Gelder IC, Hagens VE, Bosker HA, et al. A comparison of rate control and rhythm control in patients with recurrent persistent atrial fibrillation. N Engl J Med. 2002;347:1834-40.

3. Carlsson J, Miketic S, Windeler J, et al. Randomized trial of ratecontrol versus rhythm-control in persistent atrial fibrillation: the Strategies of Treatment in Atrial Fibrillation (STAF) study. J Am Coll Cardiol. 2003;41:1690-6.

4. Roy D, Talajic M, Nattel S, et al. Rhythm control versus rate control for atrial fibrillation and heart failure. $\mathrm{N}$ Engl $\mathrm{J}$ Med. 2008;358:2667-77.

5. Tikosyn ${ }^{\circledR}$ [package insert]. New York: Pfizer Laboratories Div Pfizer Inc.; 2016.

6. Jaiswal A, Goldbarg S. Dofetilide induced torsades de pointes: mechanism, risk factors and management strategies. Indian Heart J. 2014;66:640-8.

7. "Hospital Adjusted Expenses per Inpatient Day by Ownership." State Health Facts. The Henry J. Kaiser Family Foundation, 2015. Web. 11 Mar. 2017.

8. "About the Milwaukee VA Medical Center." Milwaukee VA Medical Center. U.S. Department of Veterans Affairs, 2017. Web. 18 Apr. 2017.
9. Kober L, Bloch Thomsen PE, Moller M, et al. Effect of dofetilide in patients with recent myocardial infarction and left-ventricular dysfunction: a randomised trial. Lancet. 2000;356:2052-8.

10. Pederson OD, Bagger H, Keller N, Marchant B, Kober L, TorpPederson C. Efficacy of dofetilide in the treatment of atrial fibrillation-flutter in patients with reduced left ventricular function: a Danish investigations of arrhythmia and mortality on dofetilide (DIAMOND) substudy. Circulation. 2001;104:292-6.

11. Aktas MK, Shah AH, Akiyama T. Dofetilide-induced long QT and torsades de pointes. Ann Noninvasive Electrocardiol. 2007;12(3):197-202.

12. Darpo B. Spectrum of drugs prolonging QT interval and the incidence of torsades de pointes. Eur Heart J Suppl. 2001;3(suppl_K):70-80.

13. Singh S, Yellen L, Brodsky MA, Feld GK, Berk M, Billing CB Jr. Efficacy and safety of oral dofetilide in converting to and maintaining sinus rhythm in patients with chronic atrial fibrillation or atrial flutter: the symptomatic atrial fibrillation investigative research on dofetilide (SAFIRE-D) study. Circulation. 2000;102(19):2385-90.

14. Abraham JM, Saliba WI, Vekstein C, et al. Safety of oral dofetilide for rhythm control of atrial fibrillation and atrial flutter. Circ Arrhythm Electrophysiol. 2015;8(4):772-6.

15. Steinberg JS, Shah Y, Szepietowska B. Pharmacologic conversion during dofetilide treatment for persistent atrial fibrillation. Pacing Clin Electrophysiol. 2017. https://doi.org/10.1111/pace. 13055. 